\title{
Reflexiones sobre la actuación del traductor e intérprete de libras en las clases de matemática en contexto escolar inclusivo
}

Nadjara Ana Basso Morás nadjara.moras@gmail.com orcid.org/0000-0002-8683-4289 Universidade Estadual do Oeste do Paraná (UNIOESTE), Cascavel, Paraná, Brasil

Luani Griggio Langwinski luanig.lang@gmail.com orcid.org/0000-0001-8327-9147 Faculdade de Ensino Superior de São Miguel do Iguaçu (Unigraçu - FAESI), São Miguel do Iguaçu, Paraná, Brasil

Clodis Boscarioli

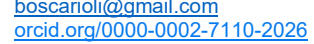
Universidade Estadual do Oeste do Paraná (UNIOESTE), Cascavel, Paraná, Brasil

\section{RESUMEN}

En Brasil, la inclusión de alumnos sordos en el contexto escolar ha sido tema de discusión desde 1990, y las barreras enfrentadas van desde la estructura de la escuela hasta la manera en que las clases son preparadas, pasando también por la mediación de un Traductor e Intérprete de Libras (TILS). Este artículo discute la dificultades encontradas por ese profesional en las clases de Matemática en el contexto escolar inclusivo, evidenciando que las escuelas, aunque con la presencia del TILS, se construyen sobre metodologías dirigidas casi que exclusivamente a los alumnos oyentes. Eso suscita algunas consideraciones relevantes acerca del papel del TILS en la transmisión del conocimiento, destacando la falta de formación inicial y continua para ese profesional. Se presenta también reflexiones y cuestionamientos para futuras investigaciones en el ámbito de la Educación Matemática Inclusiva para los alumnos sordos.

PALABRAS CLAVE: Enseñanza de Matemática para sordos. Inclusión Escolar. Traductor e Intérprete de Libras. 


\section{INTRODUCCÍON}

Los sordos conforman la comunidad sorda, o un pueblo sordo, con cultura y lengua propias. Las lenguas de señas son sus lenguas naturales, que utilizan el canal viso-espacial, y poseen estructuras gramaticales específicas, independientes de las lenguas orales de los países en que son utilizadas. En Brasil, la Lengua Brasileña de Señas (Libras) es reconocida como un medio legal de comunicación y expresión por el Decreto no 5.626/2005 que regula la Ley $n^{\circ}$ $10.436 / 2002$, que trata sobre la Libras.

La educación bilingüe en Brasil tiene como propósito básico el sordo hablar Libras con fluidez, esa es considerada su lengua natural, y como segunda lengua la modalidad escrita de la Lengua Portuguesa. Así, la educación bilingüe para sordos es considerada adecuada, pues rescata el derecho de esa comunidad a la educación, al uso de su lengua natural y al respeto por su cultura.

Actualmente en el escenario educacional de sordos, además de las escuelas bilingües, está presente la propuesta inclusiva, que ganó fuerza en los diferentes países con la Declaración de Salamanca (1994), que objetivaba fornecer directrices básicas a la formulación y reforma de las políticas y sistemas educacionales del movimiento de inclusión social y, según lo que está descrito en el documento 'Políticas de Educación Especial en la Perspectiva de la Educación Inclusiva (2008)', que versa sobre la educación de sordos en la perspectiva inclusiva, el sordo tiene el derecho al atendimiento educacional especializado.

Para la inclusión de estudiantes sordos, en las escuelas regulares, la
educación bilingüe - Lengua Portuguesa/LIBRAS -, desarrolla la enseñanza
escolar en Lengua Portuguesa y en Lengua de Señas, enseñando el
portugués como segunda lengua en modo escrito para alumnos sordos, los
servicios de traducción/interpretación de Libras y Portugués y la enseñanza
de Libras a los otros alumnos de la escuela. Se ofrece asistencia educativa
especializada, tanto en la modalidad oral y escrita, cuanto en la lengua de
señas. Debido a la diferencia lingüística, en la medida de lo posible, el
alumno sordo debe estar con otros compañeros sordos en clases regulares
en la escuela regular. La asistencia educativa especializada es proporcionada
por profesionales con conocimientos específicos en la enseñanza de la
Lengua Brasileña de Señas, de la Lengua Portuguesa como segunda Lengua,
el sistema Braille, del soroban, orientación y movilidad, de las actividades de
vida autónoma, de la comunicación alternativa, del desarrollo de procesos
mentales superiores, de los programas de enriquecimiento curricular, de la
adecuación y producción de materiales didácticos y pedagógicos, del uso de
recursos ópticos y no ópticos, de la tecnología de asistencia y otros (BRASIL,
2008, p. 16, nuestra traducción).

Aunque en los últimos años haya más referencias al respeto de la educación bilingüe para sordos, el documento ya mencionado aún propicia comprensiones ambiguas en cuanto a las lenguas utilizadas en el proceso de escolarización, suscitando el entendimiento de que el Portugués se destaca a la Libras y que el sordo disponga sólo del apoyo de un Traductor e Intérprete de Libras (TILS) durante las clases.

Nogueira y Borges (2013, p. 48, nuestra traducción), en un contexto específico, afirman que "Debemos resaltar que la escuela está pensada y organizada para una mayoría de oyentes [...]". En ese sentido, cuando se refiere a los alumnos sordos, tenemos el 'surgimiento' de una figura de extrema relevancia 
en el aspecto de la contribución significativa de enseñanza: el traductor e intérprete de Libras. Según Quadros (2004, p. 27, nuestra traducción), el traductor e intérprete de Libras es aquel "[...] profesional que domina la lengua de señas y la lengua hablada del país y que está calificado para desempeñar el papel del intérprete de Libras". Para la autora, (QUADROS, 2004, p. 27-29), el TILS es un profesional que brinda servicios como intermediario del diálogo entre diferentes culturas y costumbres. Además, él media lo que alguien dice en una lengua a otra, en otra lengua, favoreciendo la comprensión, y en este caso, él es el mediador entre el profesor, que raramente sabe Libras, y el alumno sordo.

Sin embargo, autores como Lacerda (2002), Bisol et al. (2010) y Boscarioli et al. (2018) consideran que la presencia de TILS en el aula no garantiza la igualdad de oportunidades para los estudiantes sordos, incluso en situaciones en que la formación de los TILS es satisfactoria. Para que los estudiantes sordos tengan las mismas oportunidades en un aula inclusiva, se necesita algo más, como metodologías que cumplan con las especificidades de esos alumnos, disponibilidad de materiales didácticos adecuados, asistencia pedagógica y psicológica.

Schuindt, Matos y Silva (2017) realizaron una investigación en el área de inclusión para alumnos sordos enfocada en la materia de Química y constataron que la producción de materiales en Lengua Brasileña de Señas se ha ampliado en Brasil, pero todavía hay necesidad de materiales didácticos elaborados según las características del grupo, así como su inserción en las prácticas pedagógicas inclusivas.

Ante eso, es necesario pensar sistemáticamente acerca de cómo se han llevado a cabo las clases de Matemática, en las cuales se establecen nuevas relaciones interpersonales con la presencia de los TILS en escuelas inclusivas que estudian los alumnos sordos. El objetivo de este artículo es discutir las dificultades de los TILS en las clases de Matemática en educación inclusiva, así como presentar reflexiones y cuestionamientos para la investigación en el campo de la Educación Matemática inclusiva para sordos.

\section{TRADUCTOR E INTÉRPRETE DE LIBRAS EN LAS CLASES DE MATEMÁTICA}

Aunque la Libras ha sido reconocido como un medio legal de comunicación y expresión de la comunidad sorda, la inclusión de estos individuos todavía se da de una manera complicada y problemática. En el campo educativo, la mayor discusión que involucra a la comunidad sorda es sobre la educación bilingüe y la educación inclusiva, y en el contexto de la educación inclusiva surge la figura del traductor e intérprete de Libras, que según Quadros (2004) tiene función de:

\footnotetext{
Realizar la interpretación de lengua hablada a la lengua de señas y viceversa, observando los siguientes preceptos éticos: a) confiabilidad (secreto profesional); b) imparcialidad (el intérprete debe ser neutral y no interferir con las propias opiniones); c) discreción (el intérprete debe establecer límites a su envolvimiento durante la actuación); d) distancia profesional (el profesional intérprete y su vida personal son separados); e) fidelidad (la interpretación debe ser fiel, el intérprete no puede cambiar la información porque quiere ayudar u opinar sobre algún asunto, el objetivo de la interpretación es transmitir lo que realmente se dijo) (QUADROS, 2004, p. 28, nuestra traducción).
} 
Para la autora citada, los TILS en el aula tiene la tarea de mediar la comunicación entre los alumnos sordos y oyentes y el profesor del aula, transmitiendo de manera clara y fiel el contenido escolar programado para el aula. Colaborando, Lacerda (2005, p. 366, nuestra traducción) citada por Nogueira y Borges (2013, p. 49) considera que el papel del TILS no es solo "[...] traducir contenidos, sino que hacerlos comprensibles para el alumno". La realización de esa mediación por este profesional no es una tarea fácil, por lo tanto, a lo largo del artículo haremos reflexiones sobre su actuación en el contexto escolar inclusivo.

Al comienzo del proceso educativo inclusivo para personas sordas, las prácticas de interpretación por parte de los TILS, a menudo, se realizaba de manera voluntaria. Esta práctica solo comenzó a ser reconocida efectivamente después de la aprobación de la Ley $n^{\circ}$ 12.319/2010, que estableció la profesión de TILS, eso representó una victoria, porque a partir de ella los TILS comenzaron a ser respetados como profesionales en espacios educativos públicos y privados. Según Nogueira y Borges (2013), al comienzo de ese proceso inclusivo, había una incongruencia en la formación de estos profesionales:

\begin{abstract}
Una característica importante de Brasil que "obstaculiza" la actuación de ILS es que su formación, generalmente, se lleva a cabo en cursos abiertos, de manera no profesional y se completa cuando de su actuación. Hay pocos cursos de nivel universitario para la formación de ILS y algunos de estos todavía están en la fase de implementación. Una buena parte de los profesionales que trabajan como ILS surgieron de las relaciones familiares con personas sordas y también de entornos religiosos (NOGUEIRA; BORGES, 2013, p. 48, nuestra traducción).
\end{abstract}

Silva y Fernandes (2018) también corroboran con Nogueira y Borges (2013) afirmando que incluso hoy en día una de las mayores adversidades observadas en las escuelas inclusivas está relacionada con la educación inicial y continua y, en el dominio de Libras para la actuación de los TILS. Los investigadores agregan que estos profesionales reconocen la falta de educación continua y "[...] afirman que las secretarías de educación, las instituciones públicas o privadas necesitan crear más proyectos destinados a la formación específica, ya que no poseen formación inicial en el área" (SILVA; FERNANDES, 2018, p. 44, nuestra traducción).

Además del reconocimiento de la profesión de TILS, la comunidad sorda obtuvo la implementación de la Lengua Brasileña de Señas como materia curricular en cursos de profesorado en todo Brasil, conforme el Decreto no 5.626/2005:

Art. 3ㅇ - Libras debe insertarse como una materia curricular obligatoria en los cursos de profesorado para el ejercicio de la docencia, en los niveles secundaria y universitario, y en los cursos de Foniatría de instituciones educativas públicas y privadas del sistema educativo federal. y los sistemas educativos de los Estados, el Distrito Federal y los Municipios. § 10 Todos los cursos de profesorado, en las diferentes áreas del conocimiento, el curso normal de secundaria, el curso normal superior, el curso de Pedagogía y el curso de Educación Especial se consideran cursos de formación para profesores y profesionales de la educación para el ejercicio de la enseñanza. $\S 2$ : Libras será una materia curricular opcional en los otros cursos de educación superior y en educación profesional, a partir de un año, a partir de la publicación de este Decreto (BRASIL, 2005, p. 1, nuestra traducción). 
Este Decreto $n^{\circ} 5.626 / 2005$, en su Art. $n^{\circ} 17$, también aborda la formación de TILS, señalando que esa debe hacerse a través de un curso universitario de Traducción e Interpretación, con calificación en Libras/Portugués (BRASIL, 2005). Sin embargo, aunque Libras se establece como una materia obligatoria en cursos de profesorado y capacitación específica en el área a través del curso Letras/Libras - Profesorado o Licenciatura, no es suficiente para una formación adecuada para esa categoría profesional. En la primera situación, porque una materia con una carga de horas tan pequeña que no garantiza la aptitud en Libras, además, la competencia no es el objetivo principal de esa materia, ya que Libras es una lengua y, por lo tanto, la probabilidad de adquirir una lengua en un curso de seis meses es pequeño. Y en la segunda situación, por la razón de que el número de cupos para esta capacitación es aún muy pequeño y, en la mayoría de los casos, solo se ofrece en grandes centros urbanos.

Cuando se trata de la formación de los TILS, otro factor importante a destacar es el hecho de que ellos tienen formaciones diversas y necesitan trabajar con áreas muy diferentes de su formación. Por ejemplo, un TILS pedagogo, a menudo, no está preparado para 'pasar' al alumno sordo el contenido de Matemática, porque no se trata solo de traducir, sino también de comprender los conceptos, su abstracción y aplicación, para que puedan transmitirlos, lo que requiere un esfuerzo y capacitación adicionales.

Rieger (2016) afirma que existen brechas expresadas por la distancia entre la actuación de los TILS en un aula inclusiva y su camino formativo. De acuerdo con la autora, ese "[...] distanciamiento genera dudas e inquietudes con respecto a la formación de este profesional para actuar en actividades de enseñanza, especialmente para la enseñanza de Ciencias Exactas y Naturales". Aunque hay muchas investigaciones que convergen para la Educación Matemática para sordos, todavía falta una mirada más especial a los TILS, dada la importancia de estos profesionales para la inclusión y a los procesos de enseñanza y aprendizaje del alumno sordo.

Rieger (2016), a partir del análisis bibliográfico de artículos publicados en tres ediciones del Congreso Nacional de Investigación e Interpretación de Libras y Lengua Portuguesa, considerado uno de los principales eventos científicos específicos de esa área en Brasil, mostró que:

El tema relacionado a la formación de TILS es el eje principal de discusión, se dedica poca atención a la formación del TILS para trabajar en áreas específicas del conocimiento que, en la mayoría de las veces, va más allá de su área académica de formación (RIEGER, 2016, p. 68, nuestra traducción).

Eso evidencia la falta de discusión sobre la formación básica o continua de ese profesional para la actuación en la enseñanza. Los TILS muchas veces no tienen conocimiento previo de los contenidos matemáticos que deberán traducir a los estudiantes sordos, y eso no es su culpa. Sin embargo, esa falta de capacitación puede perjudicar el aprendizaje de los estudiantes, si tienen alguna duda sobre cuál signo se debe utilizar, por ejemplo. Así, es importante que el profesor de Matemática y el intérprete mantengan un diálogo constante para minimizar esa ocurrencia y potencializar el aprendizaje de los alumnos sordos.

En esa perspectiva, hay preocupaciones por parte de la comunidad sorda de que TILS reciba una formación que les permitirá actuar con más calidad y fluidez 
en ambas lenguas, con buena traducción e interpretación, produciendo las estructuras gramaticales particulares de cada lengua, sin interferencia entre ellas. A su vez, la categoría TILS está preocupada por la calidad de la fluidez en Libras, porque sin formación inicial y continua, su actuación profesional puede verse comprometida y desagregada de las innovaciones lingüísticas de la comunidad sorda. Por lo tanto, si consideramos la falta de formación inicial y continua para el profesional de TILS, algunos problemas podrán surgir directamente relacionados con su mediación, particularmente, en las clases de Matemática.

Quadros (2004) relata que las competencias y responsabilidades de los profesores y del TILS en el aula no son tan fáciles de definir:

A menudo, el papel del intérprete en el aula termina siendo confundido con el papel del profesor. Los estudiantes dirigen las preguntas directamente al intérprete, comentan y discuten sobre temas con el intérprete en lugar del profesor. El propio profesor delega al intérprete la responsabilidad de asumir la enseñanza de los contenidos desarrollados en clase (QUADROS, 2004, p. 60, nuestra traducción).

Según Lacerda (2009, p. 73, nuestra traducción), para que esta mediación suceda satisfactoriamente debe haber cooperación entre el profesor y el intérprete, ya que "las formas de actuar del profesor interfieren en la forma de actuar del ILS, y esas cuestiones deben ser consideradas cuidadosamente para un buen progreso del trabajo escolar". Así, es necesario comprender la relación entre el profesor y los TILS, la importancia y el papel de cada uno en la educación inclusiva para sordos. Consecuentemente, es importante que el profesor y los TILS tengan en cuenta la diferencia cultural y lingüística de los sordos durante su práctica profesional, proporcionándoles actividades que contemplen experiencias visuales (ZANONI, 2016).

En ese sentido, Borges (2013, p. 60, nuestra traducción) ya argumentaba que "[...] el nivel de conocimiento de la lengua de señas del profesor de aula inclusivo, con la presencia de alumnos sordos, también influye no solo en la enseñanza y el aprendizaje de esos estudiantes, pero, incluso, en el trabajo de los intérpretes en este ambiente".

Boscarioli et al. (2015) sobre eso también relatan que:

\begin{abstract}
Considerando que el intérprete no tiene formación en el área y el profesor no domina Libras, no es posible averiguar si el concepto se pasó correctamente al alumno durante el proceso de traducción. La relación entre estos actores en el proceso de enseñanza-aprendizaje se puede resumir de la siguiente manera: el profesor y el alumno detienen conocimiento, avanzado y básico, respectivamente, sobre el contenido trabajado, pero no comparten Libras; los TILS y el estudiante comparten Libras, pero no el conocimiento básico; el profesor y los TILS comparten la lengua portuguesa, pero no el conocimiento específico (BOSCARIOLI et al., 2015, p. 8, nuestra traducción).
\end{abstract}

Además, los investigadores afirman que una de las principales dificultades que los TILS encuentran en el aula está relacionada al "[...] vocabulario de Libras que todavía es insuficiente para expresar todos los conceptos científicos" (BOSCARIOLI et al., 2015, p. 2, nuestra traducción). Estos autores enfatizan que las dificultades con los conceptos y signos de áreas específicas constituyen 
barreras lingüísticas que pueden interferir en el proceso de apropiación del conocimiento por parte del alumno sordo. Ya para Pinho (2017),

[...] el proceso de interpretación ni siempre mantiene la fidelidad del contenido al mensaje original y las barreras lingüísticas que existen en el proceso de interpretación, como la falta de signos específicos para términos técnicos y científicos y la falta de familiaridad con el tema mediado, puede dañar el proceso de enseñanza y aprendizaje del alumno sordo (PINHO, 2017, p. 8, nuestra traducción).

Las ausencias de esas señales pueden generar, en algunas situaciones, interpretaciones inadecuadas, es decir, si el TILS, por no tener formación en Matemática, al realizar la interpretación de un contenido en particular use un signo de la lengua de señas que no corresponde al término científico referido, causará daños en el aprendizaje del estudiante sordo. Corroborando esta discusión, Pinto y Vianna (2018) afirman que:

\footnotetext{
Enseñar Matemática al alumno sordo es un desafío tanto para los profesores como para los intérpretes en relación al uso de la Libras aplicado a contextos matemáticos. La juventud de la Libras en los espacios escolares y académicos implica que no siempre existen los signos necesarios para la interpretación de los términos matemáticos (PINTO; VIANNA, 2018, p. 9, nuestra traducción).
}

Si los TILS tienen dificultades o no comprenden los conceptos que están siendo trabajados, debe pedirle al profesor que le explique este concepto para que el estudiante sordo no se vea perjudicado en su aprendizaje. Así, una vez más, se destaca la complejidad del desafío que se propone al traductor e intérprete de Libras en la clase de Matemática. Para Lacerda (2002), es importante que este profesional también tenga una formación adecuada y amplia para ejercer su profesión.

[...] el intérprete necesita poder negociar el contenido con el profesor, revelar sus dudas, las cuestiones de aprendizaje del alumno y, a veces, mediar la relación con el alumno, para que se construya el conocimiento deseado. El incómodo del profesor con la presencia del intérprete puede llevarlo a ignorar al alumno sordo, atribuyendo al intérprete el éxito o el fracaso de ese alumno (LACERDA, 2002, p. 123, nuestra traducción).

Dadas esas observaciones, volvemos a la importancia de la formación de TILS, porque esta instrucción les permite tener un mejor aprovechamiento de las informaciones que surgen en el aula, ya que necesitan realizar la interpretación de una lengua hablada a una lengua de señas y viceversa, y se enfrenta, en tiempo real, con la necesidad de interpretar entre dos lenguas diferentes y con características culturales particulares.

Como ejemplo de eso, en una clase de matemática en que el profesor esté trabajando ecuaciones, al escribir en la pizarra $2 x+1=7$ y preguntar cómo se resuelve esa ecuación, qué comportamiento se espera de los estudiantes?, suponiendo que no sepan cómo resolver la ecuación presentada en el registro algebraico. Si se les pide que piensen en un número multiplicado por dos que sumado a uno resultará en siete, qué número sería ese, muchos de esos estudiantes que no pueden resolver de manera algorítmica podrían responder con la ayuda del discurso en lenguaje natural. 
Un obstáculo es el hecho de que la equivalencia referencial se separa de la congruencia semántica. Raymond Duval, autor de la Teoría de los Registros de Representación Semiótica, en una entrevista, afirma que las dificultades más profundas de comprensión son "[...] las relacionadas con los pasajes entre lengua natural y todas las designaciones y formulaciones simbólicas" (FREITAS; REZENDE, 2013, p. 11-12, nuestra traducción).

Duval (2009), explica que existe una correspondencia semántica entre los registros cuando ocurre el paso de una representación semiótica a otro sistema de manera directa, es decir, digo dos 'equis' y escribo 2x; digo 'más', escribo +, uno 1 , digo igual a siete y escribo $=7$, admitiendo que son dos representaciones semióticamente diferentes, una representación en el registro en lengua natural/materna y la otra en el registro de escrito algebraico. Desde el punto de vista de los TILS, este transmite al alumno sordo el discurso en el orden hablado por el profesor. Aun así, aunque la interpretación en Libras es literal, uno puede preguntarse: ¿entendió el alumno el significado operativo del ' $2 x^{\prime}$, que en realidad es el dos que multiplica el valor que representa la incógnita "equis"?

Suponiendo una segunda situación: el quíntuplo del número de niñas en cierto 70 año disminuido de cinco es igual a 25. ¿Cuántas niñas hay en ese 7음 año? Aunque la actividad se ocupa del contenido de una ecuación con solo una incógnita, en qué cuando se la escribe algebraicamente será muy similar a la anterior, para Duval (2012), esas representaciones no son congruentes, porque decir el quíntuplo y escribir $5 x$ son diferentes formas de referir al mismo objeto matemático y complementa que,

\footnotetext{
Dos expresiones pueden ser sinónimas o referencialmente equivalentes (ellas pueden "querer decir la misma cosa", pueden ser verdaderas o falsas al mismo tiempo) y no ser semánticamente congruentes: en este caso, hay un costo cognitivo importante para la comprensión. Entre dos expresiones, dos presentaciones de una información, hay dos relaciones independientes a considerar: la relación de equivalencia referencial y la relación de congruencia semántica. Dos expresiones diferentes pueden ser referencialmente equivalentes sin ser semánticamente congruentes. Por el contrario, dos expresiones pueden ser semánticamente congruentes sin ser referencialmente equivalentes (DUVAL, 2012, p. 100, nuestra traducción).
}

Eso puede generar otro problema para el intérprete de Libras, que en este caso podrá tener otras dificultades adicionales, ya que son objetos subjetivos que requerirán otros conceptos que el alumno sordo, muchas veces, no lleva consigo, por ejemplo, $x+x+x+x+x$ es $5 x$, es decir, tenemos cinco veces la 'equis', $y$ esta "equis" puede ser solo una letra del alfabeto que aparece cinco veces en una suma. Sin embargo, cuando tenemos el enunciado "El quíntuplo del número de niñas en un cierto 70 año disminuido de cinco es igual a $25^{\prime \prime}$, que está representado algebraicamente por $5 x-5=25$, la 'equis' es una incógnita que representa la solución de la ecuación, que debe ser encontrada por el alumno. Además, las operaciones de suma y multiplicación tienen diferentes costos cognitivos. A partir de ese hecho, se puede esperar que el intérprete al hacer la señal de quíntuplo termine haciendo la señal $5 x$ o incluso resolviendo para el alumno, como lo atestiguan Nogueira y Borges (2013).

El estudio de la Matemática puede ser menos o más difícil para el alumno sordo, dependiendo de las concepciones de esa enseñanza, de la presentación del contenido impartido por el profesor y también de la familiaridad del TILS con 
la materia. Otro ejemplo, presentado por Pinto y Vianna (2018), está relacionado con el uso excesivo de la dactilología:

\begin{abstract}
En medio de un extenso discurso del profesor que explicaba las operaciones polinómicas, la dactilología era lenta y compleja en una profusión de señales operativas que se referían a "x" e "y" y operaciones de suma, producto, división y potencia. Establecer un signo sería esencial, pero necesitaría el apoyo del profesor para ser representativa para el alumno sordo. Hay otro punto que merece destacarse: definir un signo en el aula para un término matemático, como polinomio, por ejemplo, es solo una definición local. Si los alumnos cambian de clase o de intérpretes, dependiendo de la comunicación entre sus profesores, podrán tener otro signo para ese término, lo que puede comprometer la generalización necesaria para el estudio de objetos matemáticos o dificultar la transposición de los estudios de un año escolar a otro debido a la discontinuidad de la adopción del signo (PINTO; VIANNA, 2018, p. 10, nuestra traducción).
\end{abstract}

Estas dificultades no son exclusivas de los estudiantes sordos, ya que también están presentes en la comprensión de los estudiantes oyentes al reconocer objetos matemáticos, ecuación de 1 을 grado y Polinomios, tanto en forma de lenguaje algebraico como su equivalente en lenguaje natural y con un mayor grado de dificultad para pasar del lenguaje natural a su equivalente semántico en lenguaje algebraico. Si para el alumno oyente se destacan estas dificultades, para el intérprete en el primer caso, solo hay traducción del discurso del profesor en el orden en que está hablando. En el segundo ejemplo, hay que traducir la Lengua Portuguesa para la lengua de señas de manera que el alumno escriba y resuelva en lenguaje matemático la ecuación solicitada . Y, en el caso presentado por Pinto y Vianna (2018), la complejidad de la elaboración o la ausencia de signos.

Frizzarini y Nogueira (2014) relatan el entrelazamiento entre la Libras y el lenguaje algebraico, en el que uno de los elementos centrales es el lenguaje algebraico del sordo que habla Libras con fluidez, educado en una escuela específica. Según ellas:

[...] ni todos los detalles de la Matemática están directamente relacionados con el lenguaje común y lo mismo se puede pensar de la Matemática en relación con la Libras, en que una no reemplaza a la otra, pero ambas coexisten y se basan en la misma base: la representación (FRIZZARINI; NOGUEIRA, 2014, p. 376, nuestra traducción).

Aun considerando a esas autoras, en la enseñanza del álgebra:

[...] no deberíamos separar la representación algebraica de los otros registros, debido a la necesidad de que estos estudiantes realicen una representación más que la de los oyentes. Las representaciones mentales de los sordos dependen exclusivamente de su lenguaje natural, la Libras, para generalizar y abstraer las representaciones algebraicas, teniendo los gráficos como su representación intermedia. Por eso, los alumnos sordos deben trabajar con las ventajas que les ofrece la Libras, desvinculándose del uso excesivo de algoritmos, de representaciones exclusivamente simbólicas y, sobre todo, de la obligación de obtener una respuesta apenas numérica o del lenguaje algebraico (FRIZZARINI; NOGUEIRA, 2014, p. 388, nuestra traducción).

Ellas refuerzan que esa advertencia también sirve para los alumnos oyentes, pero debe intensificarse cuando hay un estudiante sordo en el aula. Según Zanoni 
(2016), uno de los desafíos que enfrentan los alumnos sordos es el de comprender el lenguaje matemático, por su acumulación de significados en sus símbolos, códigos y representaciones, ya que están traducidos del portugués a la lengua de señas y debido a la falta de signos matemáticos, que en última instancia limita la comprensión de los conceptos estudiados. A ese respecto, Rieger (2016), señala en su investigación que faltan acciones para amparen el TILS, especialmente en contenidos específicos de las Ciencias Exactas, con el objetivo de mejorar su actuación junto a las instituciones educativas, lo que impacta en la mejora de la calidad de la enseñanza por ella ofrecida.

La ausencia de diferentes representaciones, esquemas y dibujos vinculados a las explicaciones, que a veces pasa desapercibido por el profesor, ya que muchas veces sus clases son elaboradas pensando solo en los alumnos oyentes, lo que termina perjudicando a los TILS cuanto a la interpretación y al alumno sordo cuanto a la comprensión del contenido que se está enseñando. El alumno sordo es un individuo visual.

Sin embargo, es importante tener en cuenta que esta habilidad necesita ser
estimulada, no es innata a los sordos. Es más bien un potencial, algo que los
sordos pueden hacer. No obstante, para que las imágenes que los sordos
ven sean interpretadas como conocimiento y, por lo tanto, contribuyan a su
desarrollo cognitivo, es necesario que las actividades dirigidas a la
visualización sean exploradas en el aula (PINTO; VIANNA, 2018, p. 4, nuestra
traducción).

Con todo, cabe al profesor tener ese cuidado, hablar despacio, relacionar el habla y la figura, explicar frente al alumno y no de espalda. Esas pequeñas actitudes comportamentales cooperan con el trabajo de los TILS y ayudan en la comprensión tanto de los alumnos sordos como a la de los oyentes.

Los ejemplos dados son diferentes maneras de representar el mismo objeto matemático: ecuaciones, pero, la enseñanza tradicional de la Matemática, como afirman Nogueira y Zanquetta (2008), evidencia una preocupación del profesor para transmitir a los alumnos las reglas, las definiciones, técnicas y procedimientos brevemente, sólo para 'cumplir con el programa', en el que "[...] prácticamente no existe algún trabajo con las ideas matemáticas que brinda a los alumnos un aprendizaje con la comprensión" (NOGUEIRA; ZANQUETTA, 2008, p. 233, nuestra traducción).

Se puede notar que hay una subjetividad intrínseca que puede venir en la presentación de conceptos matemáticos por parte del intérprete, o sea, que él aprendió correctamente o no, como estudiante, ya que estos conceptos son verdades variables a lo largo de la construcción de la Matemática. Un ejemplo considerable son las reglas de señales o conjunto de señales, en operaciones matemáticas, que difieren cuando se trata de operaciones de suma y resta, de las operaciones de multiplicación y división. Es evidente que este problema no es exclusivo de los estudiantes sordos, por estar también está presente en las explicaciones dadas a los estudiantes oyentes, ya que el objeto matemático sólo se deja reconocer por su representación, especialmente cuando se trata de las ecuaciones, en las cuales trabajamos el concepto de operaciones inversas.

Para Duval (2009), solo es posible que los sujetos en la fase de aprendizaje comprendan la Matemática cuando consiguen percibir la diferencia entre un objeto de su representación. Por ejemplo, los números son objetos matemáticos 
que pueden ser representados en formas decimales, fraccionarias y otras, mientras las funciones son objetos matemáticos que se pueden presentar en lenguaje natural, en lenguaje algebraico o como un gráfico. El mismo objeto matemático puede ser presentado por representaciones muy diferentes.

Las ideas del objeto matemático se forman a través de sus representaciones. Cada registro de representación describe algunas propiedades conceptuales de los objetos matemáticos por él representado. Para Duval (2009, p. 15), la aprehensión en matemática ocurre en el encuentro de la semiosis (representación) con noesis (conceptualización). Las representaciones semióticas se entienden como producciones constituidas por el uso de signos, utilizados para expresar, objetivar y tratar representaciones. El uso de representaciones semióticas no solo es esencial para fines de comunicación, sino también es necesario para el desarrollo de la actividad Matemática (DUVAL, 2009).

Aún para Duval (2009), usar los diferentes registros de representación presupone tres actividades cognitivas, a saber: formación - consiste en la selección de caracteres y determinaciones con las que se representarán los objetos, por ejemplo: el concepto de ecuación, estrictamente, vinculado a la incógnita y el signo de igual; tratamiento - transformar una representación en el mismo registro implica la manipulación algebraica y el encuentro del resultado de la ecuación; y conversión - transformar una representación en otro registro. Como en el ejemplo dado anteriormente, en la segunda situación, "El número quíntuplo de niñas en un cierto séptimo año disminuido de cinco es igual a 25", en el que es necesario transformar el registro en un lenguaje natural para el registro algebraico.

Como se ha afirmado, los objetos matemáticos pueden ser representados por miedo de la lengua natural escrita, forma algebraica, gráficos, tablas y figuras. Pensando en un aula de Matemática inclusiva para sordos, nos deparamos con otra forma de representación, lengua de señas, y debe tenerse en cuenta el hecho de que el intérprete termina teniendo una gran influencia en la forma en que se presentan los conceptos matemáticos a los alumnos, y debemos tener en mente que la Matemática presenta objetos que existen sólo en ella propia,

[...] el concepto matemático está cargado de informaciones, que a menudo cuando se lo traduce a la lengua de señas, el traductor e intérprete se vale de las palabras cotidianas que no poseen un significado compatible para enseñar conceptos matemáticos, haciendo con que la representación en Libras sea insuficiente y obstaculizando la comprensión en matemática por personas sordas (ZANONI, 2016, p. 70, nuestra traducción).

Inferir que tales objetos, que existen sólo en Matemática, se vuelven bastante dependientes de una introducción adecuada que muchas veces va más allá del campo de la lengua de señas, implica decir que muchas veces los conceptos matemáticos son presentados a los sordos de diferentes maneras, a través del intérprete. Eso no es necesariamente malo, pero puede tener una serie de consecuencias para el aprendizaje, como la comprensión equivocada del concepto y / o contenido que se ha enseñado, la distorsión del significado etc.

Frizzarini (2014) afirma que el estudio del álgebra, para alumnos sordos, debe realizarse con las ventajas que ofrece la lengua de señas, desvinculado del uso excesivo de algoritmos y solo representaciones simbólicas para obtener solo respuestas numéricas o algebraicas. Los educadores matemáticos coinciden en 
que el contenido de álgebra es igualmente difícil para los alumnos sordos y oyentes, especialmente cuando ocurre un paso precipitado de un estudio puramente aritmético a algebraico, como sucede a menudo.

Frizzarini (2014, p. 223, nuestra traducción) señala que "[...] cuando el campo de estudio es Álgebra, con diferentes significados para las letras, el uso de diferentes registros de representación se volvía indispensable para los usuarios sordos de Libras". La coordinación de diferentes registros es esencial no solo en la Matemática en sí, pero en el conocimiento de la lengua natural, favoreciendo la escritura de textos cohesivos, elaborados y argumentativos; por la compresión de lecturas y la capacidad de colectar datos para resolver una cuestión, permitiendo al alumno ver situaciones en contextos relacionados con la vida cotidiana y en otros campos del conocimiento.

\section{CONSIDERACIONES FINALES}

En un aula inclusiva es esencial a los sordos la presencia de TILS, porque es a través de este profesional capacitado y competente en Libras es que se establece la mediación entre la comunicación de los sordos con los colegas oyentes y profesores, asegurando que reciban educación en su lengua natural. Por lo tanto, es importante enfatizar que uno de los roles de la escuela inclusiva es poner a disposición instrumentos para que ese profesional haga su trabajo de una manera que permita una mediación comunicativa efectiva.

Si analizamos las investigaciones relacionadas con la enseñanza y el aprendizaje para el alumno sordo y la actuación de los TILS en el contexto inclusivo, es común encontrar la preocupación con la formación de ese profesional, teniendo en cuenta que "esta [...] actividad abarcó profesionales de diferentes áreas, como: pedagogos foniatra y pastores, entre otros. [...] por el envolvimiento que poseen con los grupos y/o organizaciones de sordos" (ROSA, 2006, p. 78, nuestra traducción).

Son unánimes los resultados aportados por la literatura sobre la falta de una formación inicial y continua para los TILS que respalde su actuación en el aula. Sin embargo, es relevante señalar que el profesor de Matemática es el responsable del aprendizaje del alumno sordo y no el traductor e intérprete de Libras. Los TILS, en la mayoría de las veces, no posee los conocimientos matemáticos necesarios para ayudar al alumno. Así, es necesario que el profesor tenga conocimiento de la cultura sorda y de la lengua de señas, y también reconozca las especificidades de sus alumnos, en el sentido de que necesitan de representaciones a partir de sus experiencias visuales, asegurando a los alumnos sordos la igualdad de oportunidades en las clases de Matemática.

Es necesario debatir sobre una escuela verdaderamente inclusiva que construya una enseñanza de buena calidad para los alumnos sordos. Y, pensar en la formación de los TILS y desarrollar una formación educativa para esos profesionales que considere la diferencia cultural y lingüística de los sordos es un paso importante, dada la importancia y la colaboración de ese profesional en la propuesta de educación inclusiva.

Aunque la regulación de la profesión de intérprete de Libras y su actuación en el contexto escolar aún es reciente, se comprende que hay una necesidad 
urgente de acciones que los amparen, específicamente en la formación en Ciencias Exactas. Por lo tanto, el dominio de Libras es esencial para que los TILS desarrollen una mediación pedagógica con dominio lingüístico y del léxico del campo en el que actuará.

Actualmente, en Brasil, la formación del TILS ya está ocurriendo, tanto a nivel de la secundaria como a nivel universitario, ya sea una licenciatura (como el curso de Letras/Libras) o un posgrado de Lato Sensu. Lacerda (2007, p. 9, nuestra traducción) enfatiza que dicha formación debe ir más allá del aprendizaje de las lenguas y debe ser "[...] una formación plural e interdisciplinaria, visando su tránsito en la polisemia de las lenguas, en las esferas de la significación y en las posibilidades de actuación frente a la difícil tarea de traducción/interpretación". También se hace necesario viabilizar la interacción de profesionales de la escuela con profesionales con fluidez, con experiencia en el área de traducción e interpretación de Libras para transmitir teorías, intercambiar conocimientos y experiencias.

Autores como Zanoni (2016) y Pinto y Vianna (2018) destacan la importancia de repensar las metodologías utilizadas en la enseñanza de los alumnos sordos. El sordo habla por medio de sus manos y escucha con los ojos. Así, el profesor puede proporcionar actividades que exploren todos los sentidos, como el uso de recursos visuales, el uso de tecnologías digitales, cada vez más presentes en la vida cotidiana de los alumnos sordos, y de diferentes registros matemáticos que también favorecen al alumno oyente. El uso de materiales manipulables puede producir nuevos significados en la materia de Matemática. Pero, desafortunadamente, en la práctica, el uso de materiales manipulables aún no es tan común en las escuelas regulares, especialmente en los años finales de la escuela primaria y en la secundaria.

Sabemos que no es simple ni fácil para el profesor y para los TILS repensar las metodologías que involucran el uso de tecnologías, de materiales manipulables y otros recursos, para proporcionar clases de matemática inclusivas, pero el primer paso que se debe tomar está directamente relacionado con la relación entre profesor/TILS, profesor/alumno sordo, TILS/alumno sordo, de modo que el acercamiento entre estos tres actores puede contribuir para minimizar las barreras enfrentadas por el alumno sordo. Comprendemos la necesidad de investigaciones que apoyen a los profesores y los TILS para una Educación Matemática de buena calidad.

A partir de lo que exponemos, tenemos inquietudes tales como: ¿Qué puede hacer el profesor de matemática para colaborar con el trabajo de los TILS? ¿Qué los TILS esperan como formación continua relacionada con la materia de Matemática? Y pensando en la realidad inclusiva para los alumnos sordos en el campo educativo, surgen otras preguntas con respecto al traductor e intérprete de Libras. Una de ellas es: ¿de qué manera este profesional intermedia las relaciones entre profesores y alumnos, así como entre colegas sordos y oyentes en las clases de matemática?

Por fin, inferimos que el profesional de TILS va más allá del papel de la interpretación en el aula, y frecuentemente transita entre el campo de la traducción y el campo pedagógico, lo que genera críticas con respecto a la falta de formación específica. Así, vale la pena reflexionar sobre cómo la investigación científica puede contribuir [y ha contribuido] a la realización de una nuevo hacer 
de este profesional que diariamente necesita ser el agente de paso de una lengua a otra, sin olvidar que la responsabilidad de la enseñanza pertenece al profesor, pero que, las circunstancias en el aula requieren que el TILS adopte una postura que vaya más allá de esta idea, lo que lo lleva a actuar como facilitador en la comunicación y la enseñanza. 


\title{
Reflections on the performance of the translator and interpreter of pounds in mathematics classrooms in the inclusive school context
}

\begin{abstract}
In Brazil, the school inclusion of deaf students has been a subject of discussion since 1990, and the barriers faced range from the structuring of the school to the way which the classes are prepared, also through mediation by the Translator e Interpreter of Libras (TILS). This paper discusses the difficulties encountered by this professional in mathematics classes in this inclusive school context, evidencing that schools, even with the presence of the interpreter, are built on methodologies that are almost totally focused on the listening students, which carries with it some relevant considerations about the role of TILS in the transmission of knowledge, highlighting the lack of initial and continuing training for this professional, as well as presenting reflections and questions for future research in the field of inclusive mathematics education for deaf students.
\end{abstract}

KEYWORDS: Mathematics education for Deaf. School Inclusion. Translator and Interpreter of Libras. 


\section{NOTAS}

1. Strobel (2015, p. 38, nuestra traducción) se reporta a pueblo sordo cómo "los sujetos sordos que no habitan el mismo local, pero que están conectados por un origen, por un código ético de formación visual, independiente del grado de evolución lingüística, tales como la lengua de señas, la cultura sorda y cualquier relación".

2. En este trabajo, se utiliza la denominación Traductor e Intérprete de Libras (TILS), aunque haya otras denominaciones también usualmente empleadas, con intérprete de lenguas de señas (ILS), traductor/intérprete de lenguas de señas, intérprete, profesor-intérprete, profesor bilingüe, entre otras.

3. La palabra ecuación deriva del origen latina que generó las palabras "igual" y "igualdad" (GARBIN, 2009, nuestra traducción).

4. El término Registro fue elegido por Durval para distinguir los sistemas semióticos utilizados en matemática y otros sistemas semióticos utilizados fuera de la matemática.

5. Duval cuando se refiere al lenguaje natural o materna se refiere al lenguaje propia de cada local, por ejemplo, portugués, inglés, francés. En el caso del sordo, la lengua materna es la Libras.

6. Os objetos matemáticos são ideias, conceitos, propriedades, estruturas e relações, ou seja, são abstrações (DUVAL 2004, 2009).

7. “[...] se atribuye al sujeto un papel irrelevante en la elaboración y adquisición del conocimiento. Al individuo que está adquiriendo conocimiento compete memorizar definiciones, enunciados de leyes, síntesis y resúmenes que les son ofrecidos en el proceso de educación formal a partir de un esquema atomístico" (MIZUKAMI, 1986, p. 11, nuestra traducción).

\section{REFERENCIAS}

BISOL, C. A.; VALENTINI, C. B.; SIMIONI, J. L.; ZANCHIN, J. Estudantes surdos no ensino superior: reflexões sobre a inclusão. In: Cadernos de Pesquisa, 2010, p. 147-172.

BRASIL. Declaração de Salamanca e linha de ação sobre necessidades educativas especiais. Brasília: UNESCO, 1994.

BRASIL, Presidência da República. Decreto $n^{\circ} 5.626$ de 2005. Regulamenta a Lei no 10.436 de 2002, que dispõe sobre a Língua Brasileira de Sinais - Libras e o art. 18 da Lei $n^{\circ}$ 10.098, de 19 de dezembro de 2000. Brasília, Distrito Federal, 2005. Disponível em: http://www.planalto.gov.br/ccivil_03/_ato20042006/2005/decreto/d5626.htm. Acesso em: out 2019.

BRASIL. Ministério da educação. Política Nacional de educação especial na perspectiva da Educação Inclusiva. Brasília: MEC/SEESP, Secretaria de Educação Especial, 2008. 
BORGES, F. A. A educação inclusiva para surdos: uma análise do saber matemático intermediado pelo Intérprete de Libras. 259 f. 2013. Tese (Doutorado) - Programa de Pós-Graduação em Educação para a Ciência e a Matemática, Universidade Estadual de Maringá, Maringá, 2013.

BORGES, F. A. A mediação para surdos inclusos nas aulas de matemática por intérpretes de libras: uma ação interlínguas? In: XII Encontro Nacional de Educação Matemática, 2016. Disponível em: http://www.sbembrasil.org.br/enem2016/anais/pdf/6383_3290_ID.pdf. Acesso em: out 2019.

BOSCARIOLI, C.; GALANTE, G.; OYAMADA, MARCIO; ZARA, R. A.; VILLWOCK, R. Aluno Surdo na Ciência da Computação: Discutindo os Desafios da Inclusão. In: 23 Workshop sobre Educação em Computação, 2015. Pernambuco, Recife. Anais do WEI 2015, 2015. Disponível em: http://www.lbd.dcc.ufmg.br/bdbcomp/servlet/Evento?id=818. Acesso em: out 2019.

CONDILAC, E. B. A língua dos cálculos. In: Os pensadores: Condilac, Helvétius, Degérando. Textos escolhidos. Traduções de Luiz Roberto Monzani, Carlos Alberto Ribeiro de Moura, Nelson Alfredo Aguilar, Scarlett Z. Marton, Mary Amazonas Leite de Barros, Hélio Leite de Barros, Armando Mora D’Oliveira, Andreas Pavel, Franklin Leopoldo e Silva, Victor Knoll. São Paulo: Abril Cultural, 1979, p. 135-192.

DUVAL, R. Semiosis y pensamiento humano: registros semióticos y aprendizajes intelectuales. Universidade del Valle, Instituto de Educación y Pedagogía-Grupo de Educación Matemática, 2004.

DUVAL, R. Semióses e pensamento humano. Tradução de L. F. Levy e M. R. A. Silveira. São Paulo: Livraria da Física, 2009.

DUVAL, R. Diferenças semânticas e coerência matemática: introdução aos problemas de congruência. Trad. de Méricles Thadeu Moretti. Revemat: Florianópolis, 2012, p. 97-117.

FREITAS, J. L. M.; REZENDE, V. Entrevista: Raymond Duval e a Teoria dos Registros de Representação Semiótica. In: Revista Paranaense de Educação Matemática RPEM, Campo Mourão, PR, v. 2, n. 3, jul-dez. 2013.

FRIZZARINI, S. T. Estudo dos registros de representação semiótica: implicações no ensino e aprendizagem da álgebra para alunos surdos fluentes em língua de sinais. 2014. 288 f. Tese (doutorado - Centro de Ciências Exatas). Programa de Pós-Graduação em Educação para a Ciência e a Matemática, Universidade Estadual de Maringá, Maringá, 2014.

FRIZZARINI, T.; NOGUEIRA, C. M. I. Conhecimentos prévios dos alunos surdos fluentes em libras referentes à linguagem algébrica no Ensino Médio. In: Revista Educação Especial. Santa Maria, v. 27, n. 49, mai-ago. 2014. p. 373-390. 
GERHARDT, T. E.; SILVEIRA, D. T. Métodos de pesquisa. Coordenado pela Universidade Aberta do Brasil - UAB/UFRGS e pelo Curso de Graduação Tecnológica - Planejamento e Gestão para o Desenvolvimento Rural da SEAD/UFRGS. - Porto Alegre: Editora da UFRGS, 2009.

GOLDFELD, M. A criança surda: linguagem e cognição numa perspectiva sociointeracionista. São Paulo: Plexus Editora, 2002.

LACERDA, C. B. F. O intérprete educacional de língua no ensino fundamental: refletindo sobre limites e possibilidades. In: LODI, A. C. B.; HARRISON, K. M.P.; CAMPOS, S. R. L.; TESKE, O. (Org.) Letramento e minorias. Porto Alegre: Mediação, 2002.

LACERDA, C. B. F. O que dizem/sentem alunos participantes de uma experiência de inclusão escolar com aluno surdo. Revista Brasileira de Educação Especial, Marília, v. 13, 2007, p. 257-280.

LACERDA, C. B. F. Intérprete de Libras em atuação na educação infantil e no ensino fundamental. Porto Alegre: Editora Mediação, 2009.

LUNA, S. V. de. Planejamento de pesquisa: uma introdução. São Paulo: EDUC, 1999.

NOGUEIRA, C. M. I.; BORGES, F. A. Um panorama da inclusão de estudantes surdos nas aulas de matemática. In: NOGUEIRA, C. M. I. (Org.) Surdez, inclusão e matemática. Curitiba: CRV, 2013, p. 44-70.

NOGUEIRA, C. M. I.; BORGES, F. A. O que muda nas aulas de escolas inclusivas com a presença do intérprete de Libras. In: NOGUEIRA, C. M. I. (Org.) Surdez, inclusão e matemática. Curitiba: CRV, 2013, p. 237-255.

NOGUEIRA, C. M. I.; ZANQUETTA, M. E. M. T. Surdez, bilinguismo e o ensino tradicional de Matemática: uma avaliação piagetiana. Zetetiké. v. 16, n. 30, 2008, p. 219-237.

MIZUKAMI, M. G. N. Ensino: as abordagens do processo. São Paulo: EPU, 1986.

PARANÁ. Secretaria de Estado da Educação/ Superintendência da Educação. Instrução $n^{\circ}$ 03/2012 - SEED/SUED. Estabelece normas para atuação do profissional tradutor e intérprete de Língua Brasileira de Sinais-Libras/Língua Portuguesa - TILS nos Estabelecimentos de Ensino da Rede Pública Estadual. Paraná, 2012. Disponível em:

http://www.educacao.pr.gov.br/arquivos/File/instrucoes/instrucao0032012libras .pdf. Acesso em: out. 2019.

PARANÁ. Secretaria de Estado da Educação/Superintendência da Educação. Instrução $n^{\circ}$ 08/2016 - SEED/SUED. Estabelece critérios para o Atendimento Educacional Especializado em Sala de Recursos Multifuncionais - Surdez, Ensino Fundamental, anos finais, e Ensino Médio, nas instituições da rede pública estadual de ensino. Paraná, 2016. Disponível em: 
http://www.educacao.pr.gov.br/arquivos/File/instrucoes/instrucao082016sued.p df. Acesso em: out. 2019.

PARANÁ. Secretaria de Estado da Educação/ Superintendência da Educação.

Deliberação n 02/2016 - SEED/SUED. Dispõe sobre as Normas para a

Modalidade Educação Especial no Sistema Estadual de Ensino do Paraná, 2016.

Disponível em:

http://www.cee.pr.gov.br/arquivos/File/pdf/Deliberacoes/2016/Del_02_16.pdf. Acesso em: out. 2019.

PERLIN, G. A cultura surda e os intérpretes de sinais. In: Educação Temática Digital. Campinas, v. 7, n. 2, jun. 2006, p.135-146.

PINHO, G. C. Mediação de conceitos científicos e as barreiras linguísticas enfrentadas pelos intérpretes de LIBRAS. 2017. 79 f. Dissertação (Mestrado em Ensino) - Programa de Pós-Graduação em Ensino, Universidade Estadual do Oeste do Paraná, Foz do Iguaçu, 2017.

PINTO, G. M. F.; VIANNA, C. C. S. Interpretação em libras na aula de matemática: um desafio para o intérprete educacional de libras In: SIPEM - VII Seminário Internacional de Pesquisa em Educação Matemática. Anais do VII SIPEM, 2018. Disponível em:

http://www.sbemparana.com.br/eventos/index.php/SIPEM/VII_SIPEM/paper/vie w/584/565. Acesso em: out. 2019.

QUADROS, R. M. 0 tradutor e intérprete de língua brasileira de sinais e língua portuguesa. Secretaria de Educação Especial; Programa Nacional de Apoio à Educação de Surdos. Brasília: MEC/SEESP, 2004.

RIEGER, C. P. E. A formação de intérprete de Libras para o ensino de ciências Lacunas refletidas na atuação do TILS em sala de aula. 85 f. Dissertação (Mestrado em Ensino) - Programa de Pós-Graduação em Ensino, Universidade Estadual do Oeste do Paraná, Foz do Iguaçu, 2016.

ROSA, A. S. Tradutor ou Professor? Reflexão preliminar sobre o papel do intérprete de língua de sinais na inclusão do aluno surdo. In: Ponto de vista, Florianópolis, n. 8, 2006. p. 55-74. Disponível em: https://periodicos.ufsc.br/index.php/pontodevista/article/view/1106/904. Acesso em: out 2019.

RUIZ, A. I.; RAMOS, N. M.; HINGEL, M. Escassez de professores no Ensino Médio: propostas estruturais e emergenciais. Brasília, 2007.

SCHUINDT, C. C.; MATOS, C. F. de; SILVA, C. S. da. Estudo de caso sobre as dificuldades de aprendizagem de alunos surdos na disciplina de Química. ACTIO, Curitiba, v. 2, n. 1, jan./jul. 2017, p. 282-303.

SILVA, D.; FERNANDES, S. F. O tradutor intérprete de língua de sinais (TILS) e a política nacional de educação inclusiva em contextos bilíngues para surdos: um estudo da realidade da rede pública estadual paranaense. Revista Educação 
https://periodicos.ufsm.br/educacaoespecial/article/viewFile/24814/pdf. Acesso em: out. 2019.

STROBEL, K. L. As imagens do outro sobre a cultura surda. Florianópolis: Ed. da UFSC, 2015.

ZANONI, G. G. Uma sequência didática proposta para o ensino de funções na escola bilíngue para surdos. 2016. 263 f. Dissertação (Mestrado em Ensino) Programa de Pós-Graduação em Ensino, Universidade Estadual do Oeste do Paraná, Foz do Iguaçu, 2016.

Recibido: 15 sep. 2019

Aprobado: 03 feb. 2020

DOI: 10.3895/actio.v5n1.9936

Cómo citar:

MORÁS, N. A. B.; LANGWINSKI, L. G. BOSCARIOLI, C. Reflexiones sobre la actuación del traductor e

intérprete de Libras en las clases de Matemática en contexto escolar inclusivo. ACTIO, Curitiba, v. 5, n. 1, p.

1-20, ene./abr. 2020. Disponible en: <https://periodicos.utfpr.edu.br/actio>. Acceso en: XXX

Dirección postal:

Nadjanara Ana Basso Morás

Rua: Damião Ferreira do Nascimento, n. 080, Polo Centro, Foz do Iguaçu, Paraná, Brasil.

Direito autoral: Este artículo está licenciado bajo los términos de la Licencia Creative Commons-Atribución

4.0 Internacional.

(c) (1) 